Check for updates

Cite this: RSC Adv., 2019, 9, 6724

\title{
In vitro and in vivo evaluations of the fully porous Ti6Al4V acetabular cups fabricated by a sintering technique
}

\author{
Ji Li, (D) $\dagger^{a}$ Wei Li, $\dagger^{b}$ Zhongli Li, ${ }^{\text {*a }}$ Yuxing Wang, ${ }^{a}$ Ruiling $\mathrm{Li}^{\mathrm{c}}$, Jiangping $\mathrm{Tu}^{\mathrm{c}}$ \\ and Gong Jin ${ }^{d}$
}

A type of canine fully porous Ti6AI4V acetabular cup was fabricated by a well-controlled powder sintering technique. The traditional hydroxyapatite-coated (HA-coated) cups were also prepared as the control. The characteristics, mechanical and biological properties of the two types of cups were evaluated by scanning electron microscopy, mechanical tests, finite element analysis and canine total hip arthroplasty (THA). Results showed that the porous cup had high porosity and large pore size with good mechanical properties without obvious stress shielding, and it had sufficient safety for implantation according to the finite element analysis. Both groups showed good biocompatibility and osteogenic ability after the THA surgeries, but the porous group had more bone ingrowth and higher bone-implant contact rate according to the micro-CT and histopathologic results. Therefore, the canine fully porous Ti6Al4V acetabular cup fabricated by the sintering technique could provide sufficient space and adequate mechanical support without obvious stress shielding effect for bone ingrowth. Compared with the traditional HA-coated cup, the porous cup may be more effective in achieving in vivo stability, which could contribute to reducing the risk of aseptic loosening.

Received 24th January 2019
Accepted 21st February 2019

DOI: 10.1039/c9ra00638a

rsc.li/rsc-advances promoting the bonding of the prosthesis with osseous tissues. ${ }^{\mathbf{1 0}}$ However, related studies showed that the mechanical properties of HA are poor. ${ }^{11,12}$ Its bending strength and fracture toughness are lower and difficult to use in a load-bearing role because of coat breakage. ${ }^{\mathbf{1 0 , 1 2}}$ Furthermore, after THA, bone tissue can only extend to the surface area and not the interior of the cup, biological fixation is not achieved when long-term stability is not guaranteed. ${ }^{13,14}$ Additionally, due to the mismatch in elastic modulus (Ti6Al4V is about $115 \mathrm{GPa}$ while cancellous bone is about $0.5-2 \mathrm{GPa}$ ), the Ti6Al4V or HA coated Ti6Al4V cup causes stress-shielding after implantation in the body ${ }^{15}$ which will lead to bone resorption and eventually aseptic loosening. ${ }^{16}$

Survivorship of the acetabular component has garnered the greatest amount of attention in the generational redesign of total hip arthroplasty components. ${ }^{7}$ Porous metal has attracted interests because of its excellent biological properties in recent years. ${ }^{17}$ A certain degree of porosity reduces the elastic modulus of metal and the stiffness mismatch between bone and implant and can hence ameliorate the effects of stress shielding on bone resorption. ${ }^{18,19}$ Furthermore, porous metals allow bone growth into the pores that promote optimum osteo-integration and strengthen the bone-implant interface, eventually enhancing bone-material contact and stability. ${ }^{20}$ The surface in cementless THA has been subjected to porous surface processing to obtain bone ingrowth, ${ }^{\mathbf{1}}$ good clinical results and long-term survival rates have been reported for the porous surfaces obtained. ${ }^{21,22}$ Many techniques have been investigated to produce porous 
metallic structure, ${ }^{23,24}$ but these conventional techniques result in low porosity and high possibility of pore blockage and have very limited control of the internal pore architecture and external shape of the porous implants. ${ }^{25}$

Most current works on porous materials focus on their use as surface coating on the implant to allow bone ingrowth to achieve biologic fixation. ${ }^{26}$ However, previous studies indicated that, as a porous coating on a stiffer solid substrate, the mechanical advantages of the porous Ti6Al4V would lost, ${ }^{27}$ thus we present that the fully porous acetabular cup may be an ideal choice. In this study, a type of canine fully porous Ti6Al4V acetabular cup was designed according to the computed tomography (CT) data of dogs, and then was fabricated using a well-controlled powder sintering technique. The characterizations, mechanical tests, finite element analysis and in vivo canine THA were performed to evaluated the properties of the canine fully porous Ti6Al4V acetabular cups.

\section{Materials and methods}

\subsection{Designs of the prosthesis according to the CT data}

Twelve beagle dogs (4 females and 8 males, aged 9-13 months, weighing 12.6-17.3 kg) were obtained from the zoological animal center of our hospital. After being anesthetized by injecting $2 \%$ pentobarbital sodium solution (30 $\mathrm{mg} \mathrm{kg} \mathrm{kg}^{-1}$ ) through a vein in the forelimb, a CT was used to scan the hip joint of each dog, and the three-dimensional (3D) data of the beagles' hip joints was obtained (Fig. 1a and b). Through the analysis of the CT data and 3D modeling, the canine hip prosthesis were designed by the JingHang Biotech Co., Ltd (Beijing, China) who is a famous artificial hip prosthesis manufacturing company in China. The sizes and shapes of the prosthesis components were determined to match the canine hip anatomy.

\subsection{Preparation of porous Ti6Al4V samples and artificial prostheses}

Ti6Al4V powder was used as the starting material in this technique. The detailed procedures were described previously. ${ }^{28-30}$ Porous rod specimens of $12 \mathrm{~mm}$ in diameter and $20 \mathrm{~mm}$ in length were prepared for the mechanical tests. The hemispherical fully porous Ti6Al4V cups with three different diameters $(18,20$ and $22 \mathrm{~mm})$ and $2 \mathrm{~mm}$ thick were prepared for canine THA according to the design above. The internal surfaces of the porous cups were smoothed by the smear technique. All the porous samples were ultrasonically treated in order to remove impurities. They were ultrasonically cleaned for 5 minutes each in two successive baths, then three times for $5 \mathrm{~min}$ in ultra-pure water. After this, the porous samples were dry in air in a sterile environment.

Traditional forging and spraying processes were performed using dense Ti6Al4V alloy to fabricate the HA-coated rod specimens $(12 \mathrm{~mm} \times 20 \mathrm{~mm})$ as the control for the mechanical tests. According to the prosthetic designs above, the HA-coated cups with three diameters $(18,20$ and $22 \mathrm{~mm}$ ) and $2 \mathrm{~mm}$ thick were fabricated. The thickness of the HA coatings was about 150 $\pm 50 \mu \mathrm{m}$. Selective laser melting (SLM) technique and spraying process were performed to fabricate the HA-coated artificial canine femoral stems with different sizes to match the femoral anatomy. Then the CoCrMo alloy femoral heads and the ultra high molecular weight polyethylene (UHMWPE) liners with different sizes were prepared to couple with the cups and stems. A whole set of canine hip prostheses components were shown in the Fig. 1c. Surgical instruments suitable for the THA surgery were also prepared. All samples in this study were sterilized in ethylene oxide prior to use. A period of at least 15 days was allowed between sterilization and experiments to enable complete diffusion of ethylene oxide from the test samples.

\subsection{Characterizations and mechanical tests of the samples}

The microstructures and exterior surface topographies of the two types of cups were examined using optical microscopes. ${ }^{29,30}$ A scanning electron microscopy (SEM) (Hitachi FE-SEM SU3500, Japan) was employed to qualitatively determine the microtopography (surface morphology, pore size, and interconnectivity). The average pore diameter values were calculated. After measuring the bulk density and apparent density using Archimedes principle, the porosity of the porous cup was calculated for each sample. Although the mechanical properties of the porous Ti6Al4V rods, such as the compressive stress and elastic modulus, have been determined in our previous study, ${ }^{29,30}$ the slight differences in different batches still existed.
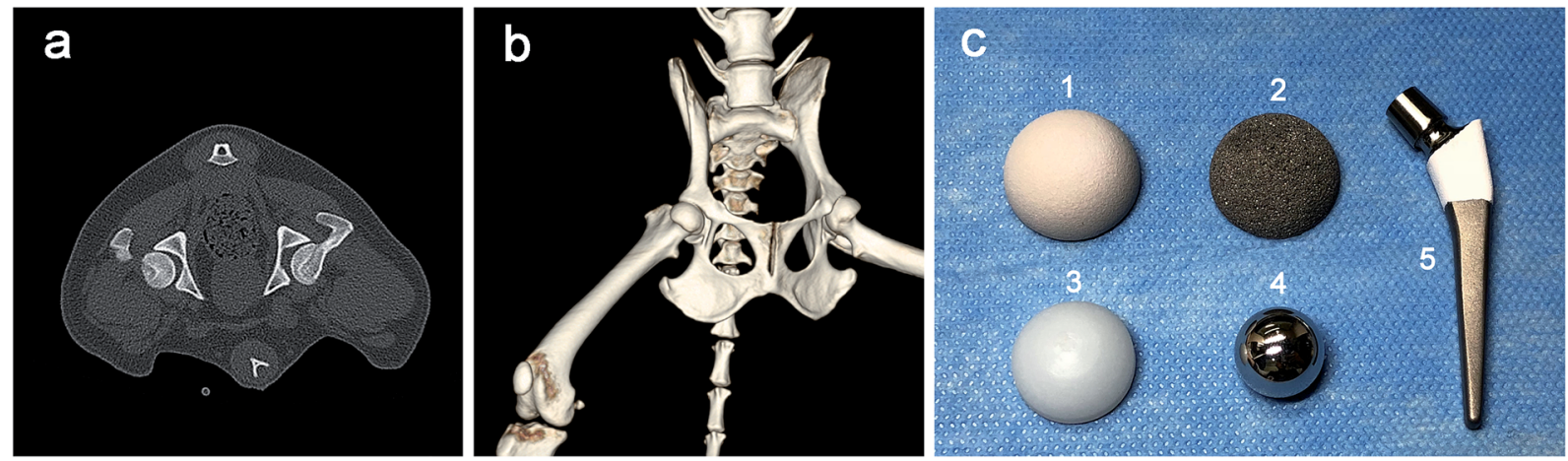

Fig. 1 CT scan and preparations of the canine prostheses: (a) CT scan showed the canine hip joint anatomic structure; (b) 3D reconstruction of the canine CT data; (c) the canine hip prostheses components: (1) HA-coated Ti6Al4V acetabular cup, (2) fully porous Ti6Al4V acetabular cup, (3) UHMWPE liners, (4) CoCrMo femoral head, (5) HA-coated Ti6Al4V femoral components. 
Thus, in order to ensure the accuracy of finite element analysis, the mechanical properties of porous samples fabricated were evaluated in this study. Axial compression tests were done to evaluate the stiffness of the two types rod samples using an MTS Alliance RT30 electromechanical test frame (MTS systems, USA). ${ }^{29,30}$ A compressive test to failure was done at a crosshead speed of $0.5 \mathrm{~mm} \mathrm{~min}^{-1}$ and the maximum load of the machine was $20000 \mathrm{~N}$, and the data of real-time compressive load versus displacement was continuously monitored and recorded. The stress-strain curves were made and then the elastic modulus was calculated for each sample.

\subsection{Finite element analysis}

To determine the mechanical safety of the canine fully porous Ti6Al4V cups in vivo, the finite element analysis was performed. According to the CT data, 3D model of the canine acetabular cup was established. Loading conditions of the standing dog were used to evaluate the mechanical response of acetabular cup in vivo, and the load on single leg is about $25 \%$ of body weight (about $38 \mathrm{~N}$ ). In this study, in order to analyze the maximum stress of acetabular cup under vertical and extrusion conditions, the load of $50 \%$ body weight (about $76 \mathrm{~N}$ ) when standing on two leg was used. Three loading modes with vertical, horizontal and slant pressures were used. In the slant mode, the acetabular cup is placed at an angle of 45 to the body's gravity line to simulate the in vivo condition after arthroplasty. Bone is treated here as isotropic material, as this simplification does not lead to a noticeable difference from those results obtained by considering orthotropic properties. $^{31,32}$ The elastic modulus and poisson ratio of fully porous Ti6Al4V cup was obtained from the mechanical test above. The elastic modulus and poisson ratio of UHMWPE liner were set as $600 \mathrm{MPa}$ and 0.46 , respectively. Binding contact is used for cup and liner. Tetrahedral elements with $0.5 \mathrm{~mm}$ side length were employed to divide the computational domain. Static, general were used in the finite element analysis, and then the Mises stress and strain of the cup under different loading conditions are calculated.

\subsection{Canine THA}

2.5.1 Animals. Twelve beagle dogs (4 females and 8 males, aged 9-13 months, weighing 12.6-17.3 kg) were obtained from the zoological animal center of our hospital. Animals were housed under standard conditions, and all surgical procedures were performed in accordance with the ARRIVE guidelines and carried out in accordance with the National Institutes of Health guide for the care and use of laboratory animals (NIH Publications no. 8023, revised 1978) and approved by Institutional Review Board of our hospital. According to a randomized implant insertion scheme, 12 beagles were divided into two groups. The fully porous Ti6Al4V cup coupled with UHMWPE liner, HA-coated Ti6Al4V stem and CoCrMo head were used for the experimental group, the HA-coated Ti6Al4V cup coupled with the same components were used for the control.

2.5.2 Surgery. Total replacement of unilateral hip joints were performed for all dogs. $2 \%$ pentobarbital sodium solution (30 $\mathrm{mg} \mathrm{kg}^{-1}$ ) was injected through a vein in the forelimb for anesthesia. The femoral trochanter was regarded as the center, and an arc incision measuring $4 \mathrm{~cm}$ in length was made. The hip joint was entered from the gap between gluteus medius and the rear lateral muscle to remove the femoral head and keep the calcar femorale within $2 \mathrm{~mm}$. Canine artificial acetabular cups were compared with the removed femoral heads to decide the appropriate size (Fig. 2a). After removal of the femoral head and neck, the soft tissue surrounding the acetabulum was retracted to allow direct observation and assessment. Preparation of the acetabular bed was performed by a cheese grater-type starter reamer to remove subchondral and cancellous bone and to achieve the appropriate depth and approximate width for the preparation. Special care was taken to ensure sufficient dorsal acetabular rim coverage and intact bone cranially and caudally to facilitate appropriate seating of the acetabular component. Following final bone bed preparation, a trial acetabular cup can be used to visually confirm the desired cup position and to confirm the cup fit with the prepared bone bed. Using an impactor instrument and mallet, the cup is driven into the bone bed until final seating and depth are achieved (Fig. $2 b$ and c). Then the UHMWPE liners were seated to couple with the cups of
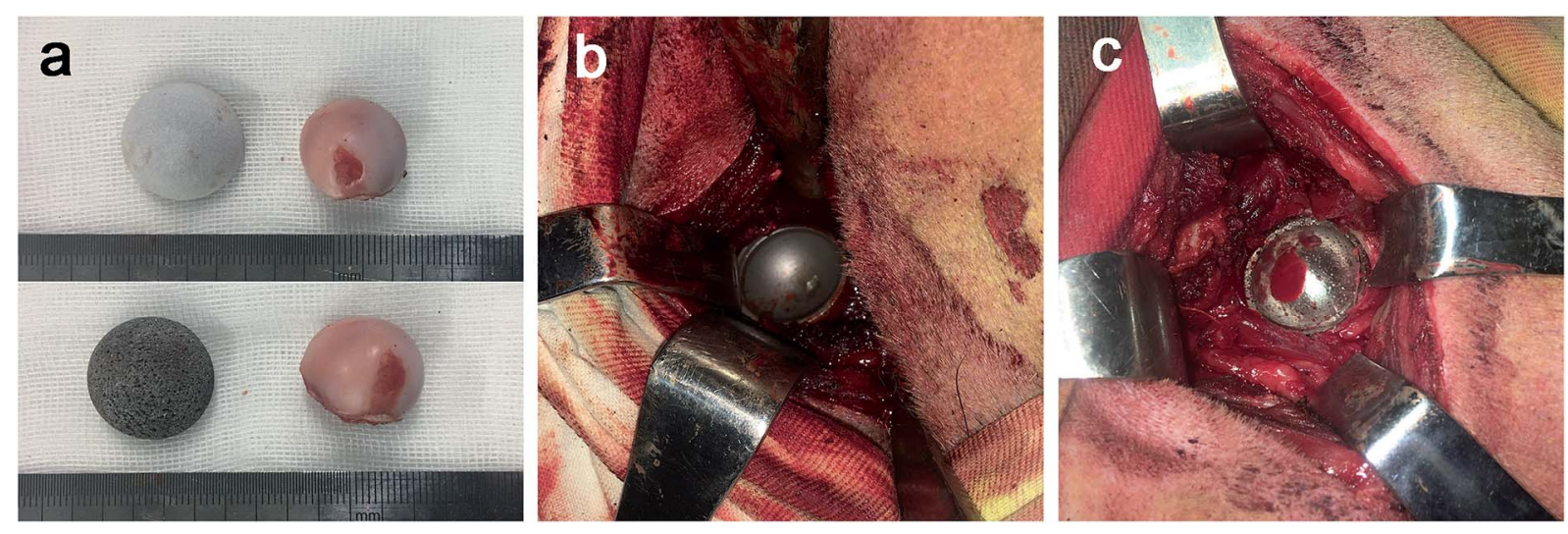

Fig. 2 Surgical procedures of canine THA: (a) canine artificial acetabular cups were compared with the removed femoral heads to decide the appropriate size; (b) implantation of the HA-coated Ti6Al4V acetabular cup in the control group; (c) implantation of the fully porous Ti6Al4V acetabular cup in the experimental group. 
same sizes. The prosthetic femur stem was inserted into the femoral medullary cavity using the self-made femoral myelocavity file. The front rake was kept between $20^{\circ}$ and $30^{\circ}$. Operative reduction was performed, and the wound was closed with layered sutures.

2.5.3 Postoperative management. After the operations, the dogs were raised separately and treated with intramuscularly $3 \mathrm{~g}$ day $^{-1}$ of penicillin for 14 days. After two weeks, the dogs were allowed to walk indoors; 3 months later, if the radiographs showed no abnormalities, then the limbs were unrestricted, and the dogs were allowed to use the limbs in voluntary outdoor activities. Then all the dogs were raised in the Experimental Animal-feeding Centre of our hospital, the outdoors activities were unrestricted every day.

Radiographs were taken for all dogs to evaluate the position of the prosthesis immediately after the surgeries, and was performed at 2, 6, 12 weeks, and 6 and 12 months postoperatively; blood sampling was performed weekly for the hematological parameters, and blood biochemistry tests were performed to monitor the dogs' health status. In addition, blood sampling was performed every two weeks for the inductively coupled plasma mass spectrometer (ICP-MS) tests to determine the metal ion content in the blood, the limit of quantification was set as $0.25 \mu \mathrm{g} \mathrm{L^{-1 }}$.

2.5.4 Micro-CT and histopathology. All the beagles were sacrificed by intra-cardiac overdose of anesthetic solution at 1 year after surgery. The cup-containing acetabulums were removed and micro-CT scanning of high resolution (Quantum GX micro-CT Imaging System, USA) was used to examine the extent of bone growth for the two groups.
After that, according to the micro-CT results, the exact implants position was identified. Then all retrieved cupcontaining acetabulums were dehydrated in a graded ethanol series $(70 \%$ to $100 \%)$ after fixation in $70 \%$ ethanol for 4 weeks. They were then embedded without decalcification in a methyl methacrylate solution, which polymerized at $37{ }^{\circ} \mathrm{C}$ within 1 week without decalcification. Then samples were subsequently cut and ground into $30 \mu \mathrm{m}$ transverse sections slices using a modified interlocked diamond saw (EXAKT, Norderstedt, Germany). After the samples were stained with hematoxylin \& eosin (HE), qualitative microscopic analyses were performed using an optical microscope attached to a digital camera. The percentage of direct contact between new bone and the implant surface (bone-implant contact, BIC) was determined by Image J software (Microsoft, Maryland, USA). The mean values for all implants in each group were calculated and subjected to statistical comparisons. Viscera (heart, liver, spleen, lung and kidney) were also retrieved and then fixed and embedded in paraffin for histopathology. Pathological sections of viscera and implant sites were evaluated macroscopically in a semiquantitative manner with respect to hemorrhage, necrosis, exudation, neovascularization cell, and encapsulation.

\subsection{Statistical analysis}

The data were analyzed by the Statistical Package for Social Sciences 17.0 software (SPSS 17.0, USA), the graphs were drawn by GraphPad Prism 5 (USA), and the results were presented as the mean \pm standard deviation (S.D.). Statistical differences were evaluated by one-way ANOVA or Student's $t$-test. $P<0.05$ was considered to indicate a statistically significant difference.
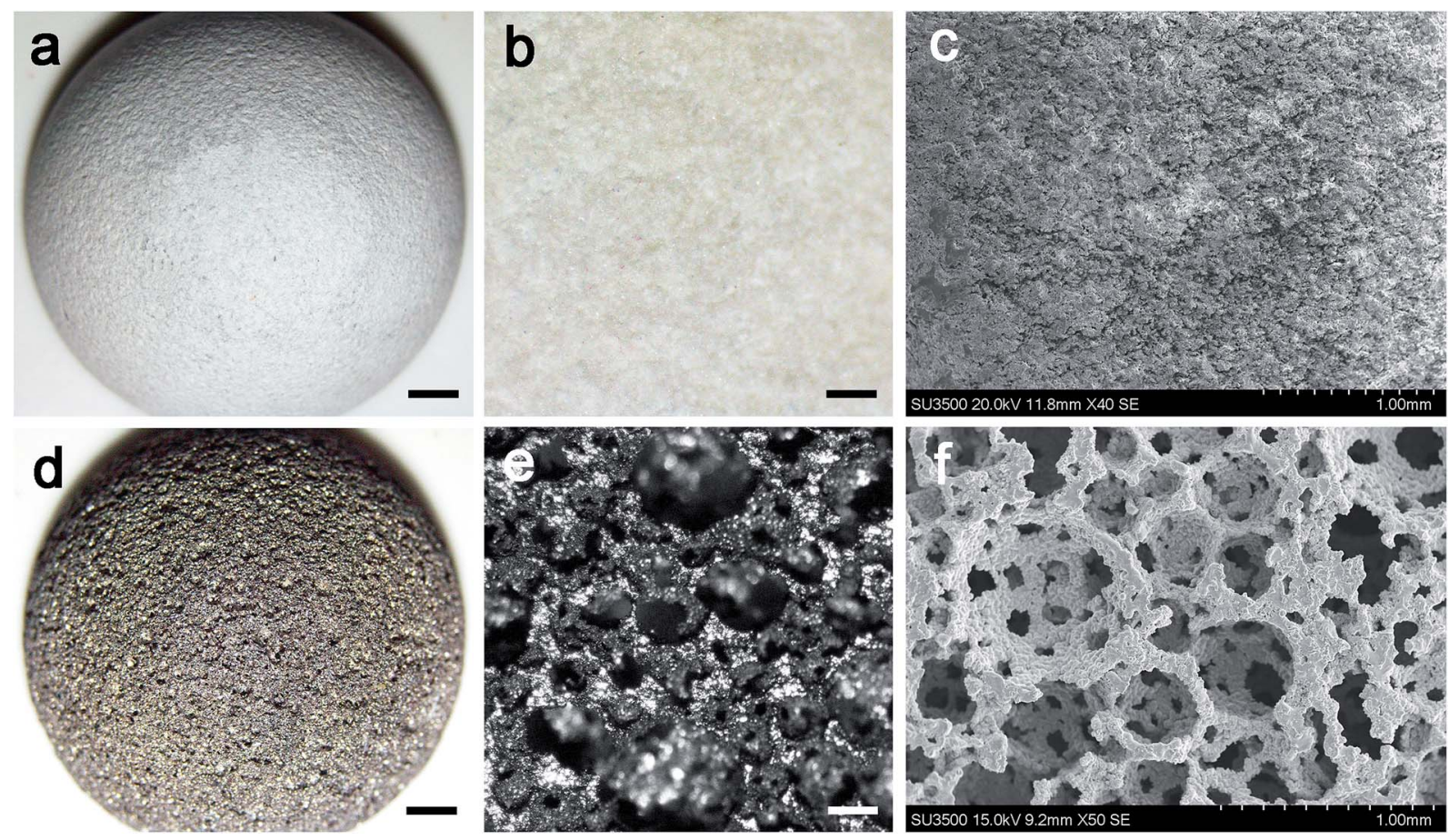

Fig. 3 Characterization of $(a-c) H A$-coated and $(d-f)$ fully porous acetabular cup: $(a)$ and $(d)$ are optical microscope images with $\times 1$ magnification, the scale bar represents $2 \mathrm{~mm}$; (b) and (e) are optical microscope images with $\times 5$ magnification, the scale bar represents $500 \mu \mathrm{m}$; (c) and (f) are SEM images, the scale bar represents $1 \mathrm{~mm}$. 


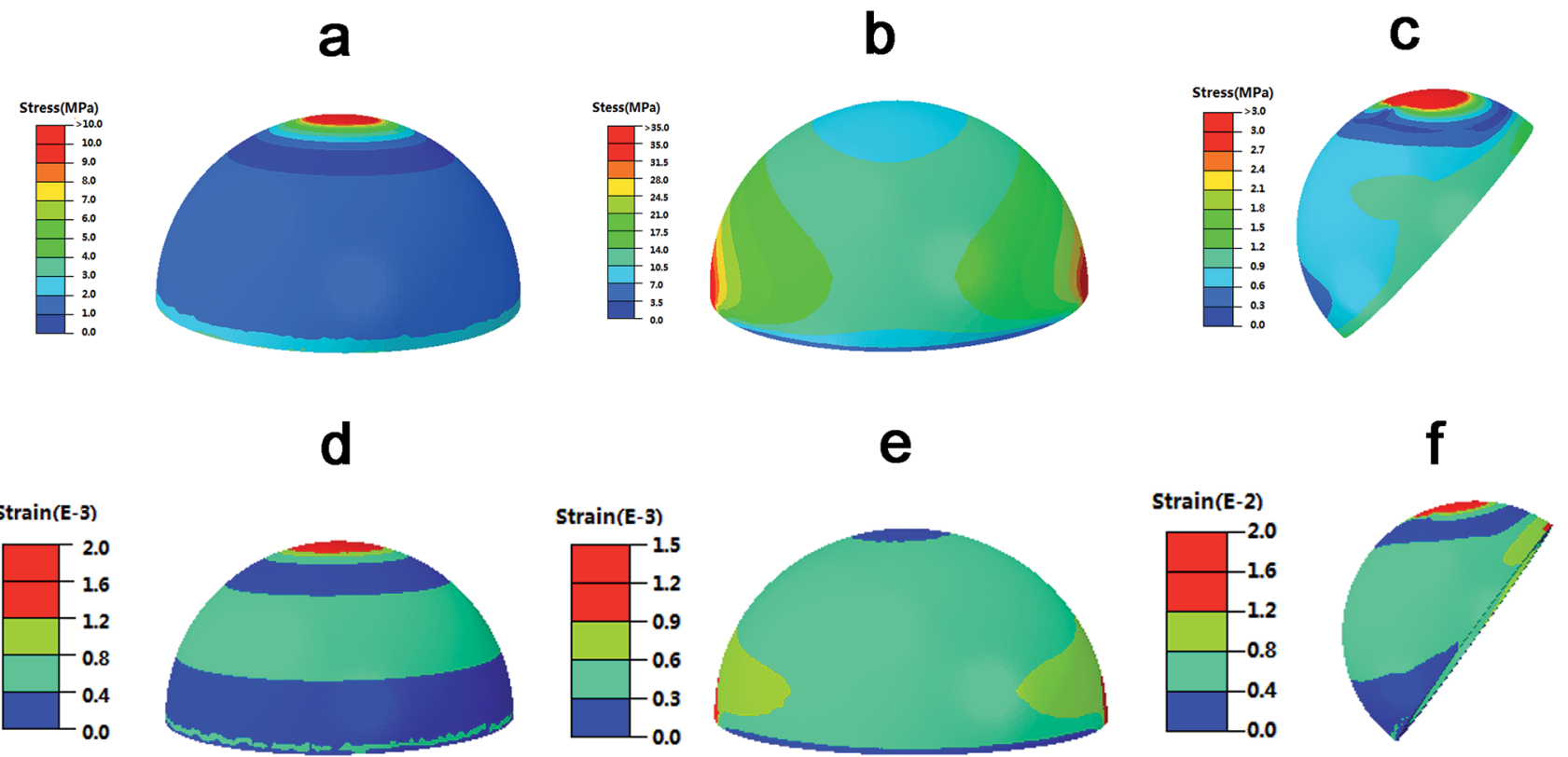

Fig. 4 Distribution nephograms of $(a-c)$ stress and $(d-f)$ strain after the finite element analysis in different loading conditions: (a) and (d) vertical pressure; (b) and (e) horizontal pressure; (c) and (f) pressure of $45^{\circ}$ inclination.

\section{Results}

\subsection{Characterizations of the samples}

According to the CT data and the design of the artificial prosthesis, the artificial canine hip joints were fabricated. Fig. 3a, b, d and e showed the optical microscopy images of the exterior face of the cups. The exterior surface of porous cup was much tougher than the HA-coated one, but the struts were well formed and continuous. The HA-coated sample showed relatively smooth surface. Detailed inspection of the SEM images for the porous cup confirmed the mechanical integrity of each cell strut with the complete formation of all the struts with neither break nor discontinuity among the elements and was the absence of loose powder particles within the cell pores (Fig. 3f). Pore morphology, distribution, and interconnectivity were also demonstrated in the SEM images. For the porous cups, a number of pores were detected and 3D connectivity between them were observed (Fig. 3f). For the HA-coated samples, the HA deposited on the Ti6AlV surface was relatively smooth and well-distributed, and there was no obvious defect (Fig. 3c). After measure and calculation, we found that the average porosity of the porous sample was about $76.08 \pm$ $2.40 \%$, and the average pore size was about $508.57 \pm 211.17 \mu \mathrm{m}$. After calculations for the collected force-displacement data, the compressive stress-strain curve was obtained, and then the elastic modulus was calculated. For the HA-coated samples, the average elastic modulus was $106.33 \pm 4.36 \mathrm{GPa}$, and the average compressive strength was $960.90 \pm 44.06 \mathrm{MPa}$. For the porous sample, the average elastic modulus was $1.96 \pm 0.26 \mathrm{GPa}$, the ductility was about $4.46 \% \pm 0.35 \%$, and the average
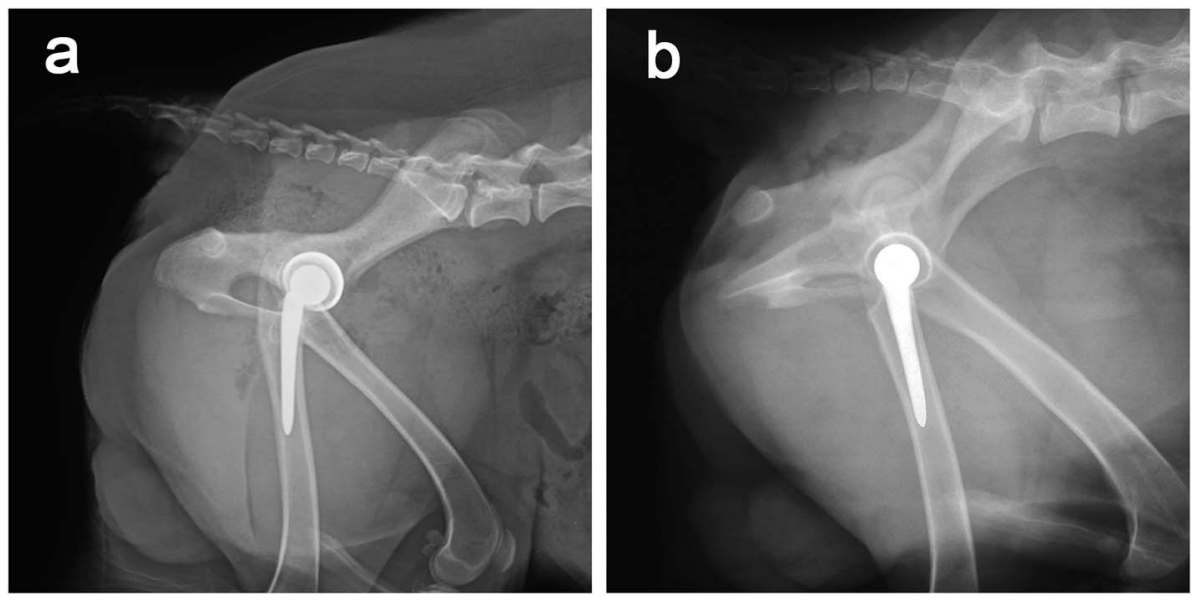

Fig. 5 Beagles' lateral radiographs of hip joints in the (a) HA-coated group and (b) fully porous Ti6Al4V group after one-year implantations. No transparent band was observed around the prosthetic cups in both groups. Slight increase in bone density was seen in the top part of the hip bone and acetabular bone. 
Table 1 The concentrations of $\mathrm{Ti}, \mathrm{Al}$ and $\mathrm{V}$ ions in the two groups ${ }^{a}$

\begin{tabular}{lllll}
\hline Ions & $\begin{array}{l}\text { HA-coated Ti6Al4V } \\
\operatorname{cup}\left(\mu \mathrm{g} \mathrm{L}^{-1}\right)\end{array}$ & $\begin{array}{l}\text { Fully porous Ti6Al4V } \\
\operatorname{cup}\left(\mu \mathrm{g} \mathrm{L}^{-1}\right)\end{array}$ & $t$ & $P$ \\
\hline $\mathrm{Ti}$ & $0.41 \pm 0.05$ & $0.45 \pm 0.10$ & -0.990 & 0.351 \\
$\mathrm{Al}$ & $0.54 \pm 0.13$ & $0.60 \pm 0.14$ & -0.742 & 0.479 \\
$\mathrm{~V}$ & $\leq \mathrm{LOQ}^{a}$ & $\mathrm{LOQ}^{a}$ & - & - \\
\multicolumn{2}{l}{${ }^{a} \mathrm{LOQ}$, limit of quantification. } & &
\end{tabular}

compressive strength was $98.55 \pm 7.65 \mathrm{MPa}$. Both of the elastic modulus and compressive strength of the HA-coated Ti6Al4V were significant higher than the porous one (both $P<0.05$ ).

\subsection{Finite element analysis}

The distribution nephograms of stress and strain in Fig. 4 showed that, in different loading conditions, the maximal stress of the fully porous Ti6Al4V cup was about $35 \mathrm{MPa}$, which was absolutely lower than the compressive stress of the sintered porous Ti6Al4V (about $98 \mathrm{MPa}$ ). The maximal strain of the cup was about 0.002 , which was absolutely smaller than the ductility of the material (about 0.04). The results indicated the mechanical responses of the fully porous cup in different loading conditions were in the scope of the compressive stress and ductility of the material. Therefore, the fully porous Ti6Al4V cup has sufficient safety to use for implantation.

\subsection{Canine THA}

The dogs showed no significant changes in food and water consumption and body weight, and no deaths or obvious clinical complications (such as infection or medicinal complications) were observed throughout the one-year follow-up. During the early postoperative period, some hematological parameters
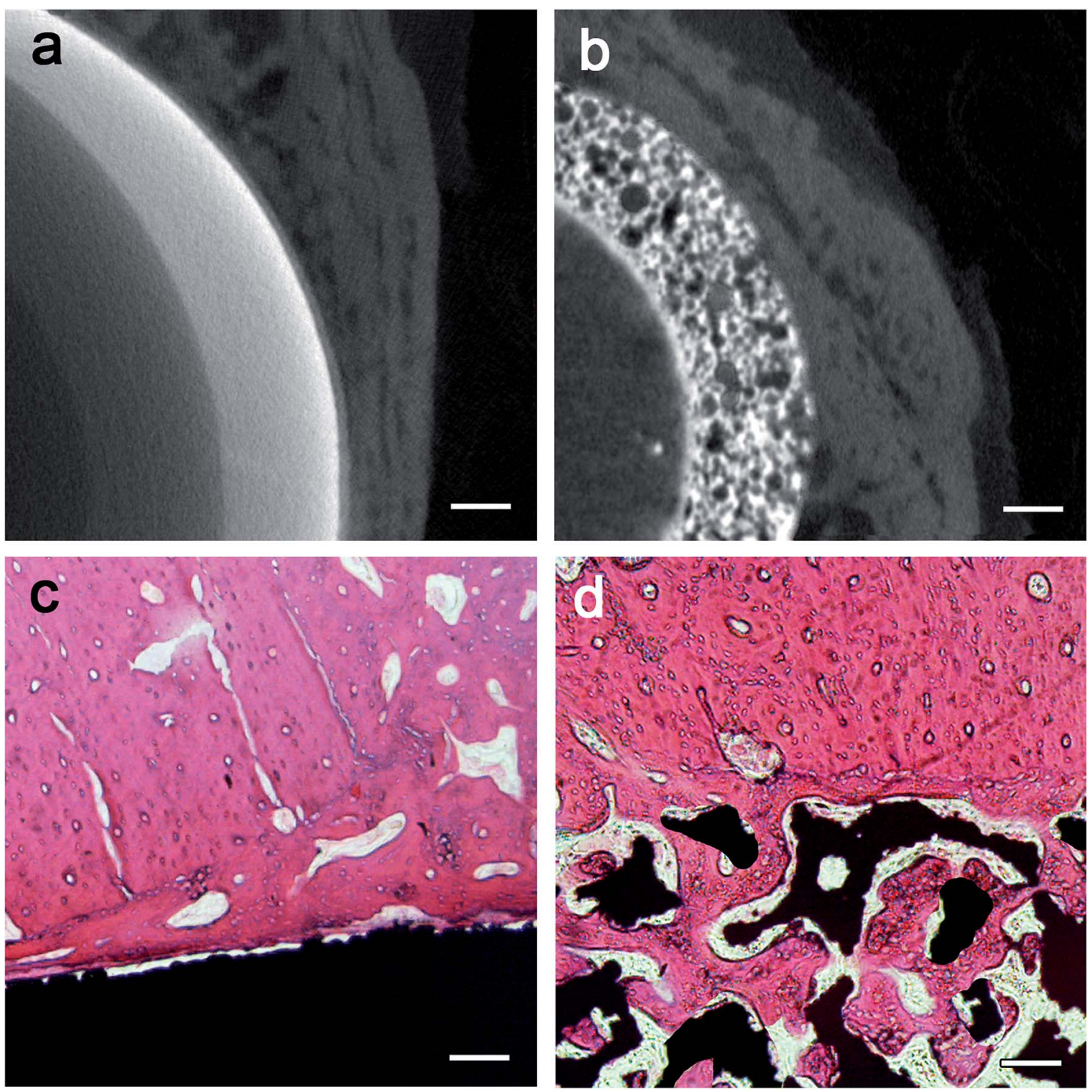

Fig. 6 Micro-CT images of (a) the HA-coated cup and (b) the fully porous Ti6Al4V after one-year implantations. The scale bar in each image represents $2 \mathrm{~mm}$. And histopathologic images of (c) the HA-coated cup and (d) the fully porous Ti6Al4V after one-year implantations. The scale bar in each image represents $200 \mu \mathrm{m}$. 
(WBC, IL-6, CRP, et al.) and blood biochemistry values (ALP, GLU, TG, et al.) changed significantly in most animals, which were attributed to a normal response to surgery. No significant differences were found in the body weight, hematology, blood chemistry and the viscera coefficient between the experimental and control animals (both female and male dogs).

3.3.1 Radiograph. At the last follow-up, radiographs showed satisfactory positions of the prosthesis, and no obvious dislocation and loosening were observed (Fig. 5). Because the exist of the amount of pores, the porous cup showed a slight higher transmittance under the X-ray than the HA-coated cup. No transparent band was observed around the prosthetic cups in both groups. Slight increase in bone density was seen in the top part of the hip bone and acetabular bone. There was no significant difference in bone density around the prostheses among the three groups.

3.3.2 Systemic metal ion analysis. For all dogs, blood sampling was performed every two weeks for the ICP-MS tests to determine the $\mathrm{Ti}, \mathrm{Al}$ and $\mathrm{V}$ ions content in the blood. Table 1 showed that, there was no significant difference of the average concentrations of $\mathrm{Ti}$ and $\mathrm{Al}$ ions between the two groups (both $P$ $>0.05$ ), the contents of the $\mathrm{V}$ ion in both groups were lower than the limit of quantification.

3.3.3 Micro-CT. The micro-CT observed a large amount of bone tissue surrounding the fully porous Ti6Al4V cups, no defects around the implant and a good bonding was observed at the interface between the bone tissue and implant interface. Images also showed that the cups were properly lodged into the acetabulum during surgery. The bone surrounding and ingrowth the implants can be observed in Fig. 6a and b where the bone volume has been clipped back in order to visualize a cross-section against the implant surface, together with the bone growth inside the porous structure. These images clearly show osseointegration in the porous network for better bonetissue bonding. In the control group, a large amount of bone tissue were also observed surrounding the HA-coated Ti6Al4V cups, but no new bone was observed to grow into the implants (Fig. 6a and b).

3.3.4 Histopathology. When incision of the canine hip joint, we found small amount of reactive synovial tissue and the prosthesis was well positioned. From the histopathologic results (Fig. 6c and d), general tissue response was similar for both groups, in no cases was inflammatory infiltration consisting of mononuclear cells (lymphocytes) observed, nor was infiltration of neutrophils and eosinophils, which demonstrates the good biocompatibility of the implants used. No osteoclastic reaction was observed in the vicinity of the implant. The histological images of the viscera samples showed that in all dogs, no abnormal alterations were found in the heart, liver, kidney, paranephros, spleen, gonad and ovary. A qualitative analysis indicated that there was no significant difference between the control and experimental groups.

The histopathologic images (Fig. 6c and d) identified the large amount of bone tissue surrounding the fully porous Ti6Al4V cups as observed by micro-CT. Bone formation was found to start from the host bone bed toward the implant in all implants. In the fully porous cup group, the majority of the pores at the bottom part of the implants were filled with bone tissue. With direct deposition of newly formed bone tissue onto the surface of the implants and ingrowth, the porous cup had almost completely integrated with the acetabulum. In the control group, new bone tissue was observed adherent to the implant, but no new bone was observed to grow into the control implants. After 1 year of implantation, the quantitative data of BIC were evaluated in the histopathologic images. Compared with the HA-coated cups $(39.87 \% \pm 4.80 \%)$, the fully porous cups $(67.70 \% \pm 5.48 \%)$ had a significant higher BIC value $(t=$ $-6.615, P=0.003)$.

\section{Discussion}

Recently, the porous metal has been applied in the design of acetabular cups. ${ }^{21,22}$ The porous metal cups always have increased surface roughness than previous cup designs, in order to provide even greater interface friction for further improved initial mechanical stability. ${ }^{7,33}$ However, the porous Ti6Al4V fabricated by the conventional technique are often very brittle and are prone to crack propagation at low stresses, ${ }^{34}$ and the pore size, shape, volume fraction, and distribution are difficult to control. ${ }^{25}$ The manufacturing of porous Ti6Al4V products is associated with difficulties, most notably the extreme chemical affinity of liquid titanium to atmospheric gases such as oxygen, hydrogen, and nitrogen, which eventually leads to strongly reduced ductility. ${ }^{19}$ Other porous metal such as porous tantalum which is excellent for its biocompatibility, high volumetric porosity, and low modulus of elasticity, but its homogenous distribution of pores has the undesirable effect of increasing interface stresses. ${ }^{35}$ Additionally, the current application of porous metal in the design of acetabular cup was as a porous coating on a stiffer solid substrate, as a result, most, if not virtually all of the advantages of its low modulus of elasticity, are lost. ${ }^{27}$

In this study, a powder sintering technique was used to fabricate the fully porous Ti6Al4V cup. By controlling the quantity and size of spacer particles added to the Ti6Al4V powder, the porous implants could be fabricated with different porosities and pore sizes to offer optimal mechanical properties and structure conditions for bone regeneration. ${ }^{36}$ Porous materials with a porosity of $30-90 \%$ and pore size of $150-600 \mu \mathrm{m}$ may be suitable for the bone ingrowth. ${ }^{\mathbf{1 3 , 1 6}}$ Although higher porosity with larger pores of porous material can provide more space for the bone to grow into, ${ }^{37}$ mechanical properties decrease rapidly with the increase in porosity. ${ }^{19}$ Thus, a balance between the microstructure and the mechanical properties was very important for a better porous implant performance. In the present study, according to the SEM images, this new type of Ti6Al4V cups showed porous structure and 3D connectivity between pores. The porosity can reach $76.08 \%$ with pore size about $508 \mu \mathrm{m}$. According to the results of mechanical tests, the porous material has high compressive strengths (98.55 Mpa) which matched that of the adult cortical bones. ${ }^{38}$ The elastic modulus value (1.96 GPa) was closer to cancellous bone, ${ }^{38}$ which was much lower than the dense Ti alloy or the HA-coated Ti6Al4V cups (about 106.33 GPa). In addition, the new sintered technique prevents the absorption 
of atmospheric gases because the processing is carried out under an inert gas or vacuum atmosphere, and with two times of slow heating, resulting in high ductility of the porous Ti alloy. Therefore, the porous Ti6Al4V material fabricated by the novel sintered technique could provide sufficient space for bone to grow into and provide adequate mechanical support and high ductility without obvious stress shielding effect. To keep the excellent mechanical responses of the porous sintered Ti6Al4V, we chose the fully porous Ti6Al4V acetabular cup design instead of the porous coatings. The results of finite element analysis showed that, in different loading conditions, the mechanical properties of cup in different loading condition were in the scope of the compressive stress and ductility of the porous Ti6Al4V material. The safety of this type cup was guaranteed for canine THA. Therefore, the canine fully porous Ti6Al4V acetabular cups with high porosity, lager pore and ideal mechanical properties were obtained.

Ti6Al4V implant was widely used in clinic around the world, it was generally safe and inert for human body. However, high dose of the metal ions released by metal implant was toxic, such as the $\mathrm{Al}$ could cause a nervous disorder and $\mathrm{V}$ could cause tissue reactions and viscera damages. ${ }^{39}$ Therefore, the ICP-MS was performed to monitor the canine systemic metal ion in this study. The results showed that there was no significant difference of concentrations of the released $\mathrm{Ti}$ and $\mathrm{Al}$ ions between the fully porous Ti6Al4V cup and the HA-coated Ti6Al4V cup, which indicated that the new cup would not increase the release of the toxic ions. The concentrations of $\mathrm{V}$ ion in both groups were much lower than the limitation of quantification, which was absolutely a safe content for human. ${ }^{40}$ The qualitative analysis of viscera histopathologic images indicated no abnormal alterations and no significant difference between the two groups. The histological results showed a tight adhesion of implant to the bone was obvious without evidence of a foreign body reaction. The general tissue response was similar for the two types of cups, and in no cases was inflammatory in filtration consisting of mononuclear cells (lymphocytes) observed, nor was infiltration of neutrophils and eosinophils, which demonstrates the good biocompatibility of the implants used. No osteoclastic reaction was observed in the vicinity of the implant. Therefore, the fully porous Ti6Al4V cups showed excellent biocompatibility.

Implant surface properties have a direct role in osteogenesis at the bone-implant interface, influencing a series of coordinated events including protein adsorption, cell proliferation, and bone tissue deposition. ${ }^{41}$ The advantage of porous materials is their ability to provide biological anchorage for surrounding bone tissues and greater contact area at the implant-bone interface. Pores with more ragged and rough surfaces could offer a larger surface area for bone in-growth..$^{16,42}$ Compared with the HA-coated cups, the 3D structure, high porosity and large pore size of the porous cups could allow more body fluids to be transported through the interconnected pores, and subsequently accelerated the healing process by allowing tissue to grow inside the implants and improved the biological fixation. ${ }^{37}$ Additionally, the rough surface of porous implant could provide solid initial stability through increased surface friction compared to the HA-sprayed designs. ${ }^{7}$ The results of histomorphometric analysis and micro-CT showed that bone grows from the host bone bed to the implants, characteristics of direct adhesion (integration) of bone to the implant are obvious, more bone ingrowth were found in the porous group. The high degree of BIC, that is, osseointegration, were found in both groups after long-term implantation in experimental animals, but statistically significant higher in the porous group. The high ingrowth values create a stable ingrowth construct while also maintaining the mechanical strength due to the integration of the porous structure and host bone bed. Our previous study also indicated that the new sintered porous Ti6Al4V was effective in achieving early stability after implantation. $^{30}$ In summary, the fully porous Ti6Al4V cups showed excellent biocompatibility and osteogenic ability. These findings indicated that the new type of cups produced by the novel sintered technique may be more effective in achieving early and long-term stability, which are very meaningful for the clinical treatments after THA surgeries. Early stability allows patients to have weight bearing and autonomous activities earlier, avoiding many bedridden risks such as thrombosis and bedsores and promoting the period of rehabilitation. Long-term stability could reduce the risk of aseptic loosening.

\section{Limitations}

There are several limitations of this study. Firstly, surface roughness of the two types of cups were not measured quantitatively. Secondly, in the finite element analysis, the mechanical responses of acetabular cup in vivo was evaluated only in the standing loading condition of the dogs, without considering the influence of muscle strength and the complex conditions when moving. Thirdly, the number of animals and the implantation time were limited, many adverse effects of the implant may not yet appear. A large number of animals with longer follow-up after implantation may be necessary to better evaluate the material's biosafety. Fourthly, the chemistry related tests were necessary to confirm the structure of the porous Ti6Al4V material, fourier transform infrared (FTIR) spectroscopy and NMR test were absent in this study. ${ }^{43,44}$

\section{Conclusions}

The canine fully porous Ti6Al4V acetabular cup fabricated by the sintered technique could provide sufficient space and adequate mechanical support without obvious stress shielding effect for bone ingrowth. Compared with the traditional HAcoated cup, the porous cup showed good biocompatibility and osteogenic ability, more bone ingrowth and higher boneimplant contact rate, which may be more effective in achieving in vivo stability. This study provide guidance for the further application of the fully sintered porous Ti6Al4V cups in human.

\section{Funding}

This work was supported by the National Key Research and Development Program (grant numbers: 2016YFC1101905). 


\section{Conflicts of interest}

There are no conflicts to declare.

\section{Acknowledgements}

I acknowledge my friend Lumeng Jin for her help with English language editing.

\section{References}

1 Y. Shinji, N. Shunji, K. Yutaka, N. Masaru, G. Tomohiro,

H. Daisuke and S. Koichi, J. Orthop. Sci., 2018, 23, 967-972.

2 C. U. Gwam, J. B. Mistry, N. S. Mohamed, M. Thomas, K. C. Bigart, M. A. Mont and R. E. Delanois, J. Arthroplasty, 2017, 32, 2088-2092.

3 J. L. Howard, H. M. Kremers, Y. A. Loechler, C. D. Schleck, W. S. Harmsen, D. J. Berry, M. E. Cabanela, A. D. Hanssen, M. W. Pagnano, R. T. Trousdale and D. G. Lewallen, J. Bone Jt. Surg., Am. Vol., 2011, 93, 1597-1604.

4 R. T. Beck, K. D. Illingworth and K. J. Saleh, J. Orthop. Res., 2012, 30, 541-546.

5 K. Corten, K. Au and R. B. Bourne, Orthopedics, 2009, 32, 6974.

6 J. L. Gaffey, J. J. Callaghan, D. R. Pedersen, D. D. Goetz, P. M. Sullivan and R. C. Johnston, The Journal of bone and joint surgery. American volume, 2004, 86-A, 257-261.

7 S. R. Small, M. E. Berend, L. A. Howard, R. D. Rogge, C. A. Buckley and M. A. Ritter, J. Arthroplasty, 2013, 28, 510-516.

8 S. A. Hacking, T. Pauyo, L. Lim, J. G. Legoux and M. N. Bureau, J. Biomed. Mater. Res., Part A, 2010, 94, 953960.

9 P. Duffy, J. L. Sher and P. F. Partington, J. Bone Jt. Surg., Br. Vol., 2004, 86, 34-38.

10 X. Zeng, J. Li, S. Yang, Q. Zheng and Z. Zou, Appl. Surf. Sci., 2012, 258, 4489-4496.

11 G. M. Vidigal Jr, M. Groisman, L. H. Gregorio and A. Soares Gde, Clin. Oral. Implants Res., 2009, 20, 1272-1277.

12 M. Roy, V. K. Balla, A. Bandyopadhyay and S. Bose, Acta Biomater., 2011, 7, 866-873.

13 W. Mróz, B. Budner, R. Syroka, K. Niedzielski, G. Golański, A. Slósarczyk, D. Schwarze and T. E. Douglas, J. Biomed. Mater. Res., Part B, 2015, 103, 151-158.

14 B. V. Krishna, S. Bose and A. Bandyopadhyay, Acta Biomater., 2007, 3, 997-1006.

15 H. Kröger, P. Venesmaa, J. Jurvelin, H. Miettinen, O. Suomalainen and E. Alhava, Clin. Orthop. Relat. Res., 1998, 352, 66-74.

16 Y. S. Amin, d. S. J. Van, Y. C. Chai, R. Wauthle, B. Z. Tahmasebi, P. Habibovic, M. Mulier, J. Schrooten, H. Weinans and A. A. Zadpoor, Biomaterials, 2014, 35, 6172-6181.

17 G. Lewis, J. Mater. Sci.: Mater. Med., 2013, 24, 2293-2325.
18 P. Heinl, L. Müller, C. Körner, R. F. Singer and F. A. Müller, Acta Biomater., 2008, 4, 1536-1544.

19 J. Parthasarathy, B. Starly, S. Raman and A. Christensen, J. Mech. Behav. Biomed. Mater., 2010, 3, 249-259.

20 J. Wegrzyn, K. R. Kaufman, A. D. Hanssen and D. G. Lewallen, J. Arthroplasty, 2015, 30, 1008-1013.

21 E. S. Vutescu, P. Hsiue, W. Paprosky and S. Nandi, Hip Int., 2017, 27, 505-508.

22 K. G. Nilsson, A. Theodoulou, G. Mercer, S. J. Quinn and J. Krishnan, J. Orthop., 2017, 14, 454-460.

23 J. Ruan, H. Yang, X. Weng, J. Miao and K. Zhou, J. Mater. Sci.: Mater. Med., 2016, 27, 1-8.

24 K. Korkmaz, Surf. Coat. Technol., 2015, 272, 72-78.

25 G. Ryan, A. Pandit and D. P. Apatsidis, Biomaterials, 2006, 27, 2651-2670.

26 N. Harrison, P. E. McHugh, W. Curtin and P. Mc Donnell, J. Mech. Behav. Biomed. Mater., 2013, 21, 37-46.

27 S. Arabnejad, B. Johnston, M. Tanzer and D. Pasini, J. Orthop. Res., 2017, 35, 1774-1783.

28 S. Mullens, I. Thijs, J. Cooymans and J. Luyten, US Pat., US8992828, 2015.

29 J. Li, Z. L. Li, R. L. Li, Y. Y. Shi, H. R. Wang, Y. X. Wang and G. Jin, RSC Adv., 2018, 8, 36512-36520.

30 J. Li, Z. Li, Y. Shi, H. Wang, R. Li, J. Tu and G. Jin, J. Mech. Behav. Biomed. Mater., 2018, 91, 149-158.

31 V. Baca, Z. Horak, P. Mikulenka and V. Dzupa, Med. Eng. Phys., 2008, 30, 924-930.

32 L. Peng, J. Bai, X. Zeng and Y. Zhou, Med. Eng. Phys., 2006, 28, 227-233.

33 K. Yoshimoto, Y. Nakashima, M. Wakiyama, D. Hara, A. Nakamura and M. Iwamoto, J. Orthop. Sci., 2018, 23, 665-670.

34 I. H. Oh, N. Nomura, N. Masahashi and S. Hanada, Mater. Sci. Eng., C, 2003, 49, 1197-1202.

35 J. H. Kuiper and R. Huiskes, J. Orthop. Res., 1996, 14, 36-43. 36 V. D. S. Johan, d. J. Van, P. Olav, S. Amin Yavari, M. F. P. De Haas, J. H. Waarsing, H. Jahr, E. M. M. Van Lieshout, P. Patka, J. A. N. Verhaar and A. A. Zadpoor, J. Orthop. Res., 2013, 31, 792-799.

37 D. Muller, H. Chim, A. Bader, M. Whiteman and J. T. Schantz, Stem Cells Int., 2010, 2011, 547247.

38 D. Yang, H. Shao, Z. Guo, T. Lin and L. Fan, Biomed. Mater., 2011, 6, 045010.

39 D. P. Perl and A. R. Brody, Science, 1980, 208, 297-299.

40 B. R. Levine, A. R. Hsu, A. K. Skipor, N. J. Hallab, W. G. Paprosky, J. O. Galante and J. J. Jacobs, J. Bone Jt. Surg., Am. Vol., 2013, 95, 512-518.

41 B. Yin, B. Xue, Z. Wu, J. Ma and K. Wang, Am. J. Transl. Res., 2018, 10, 474.

42 A. Artel, H. Mehdizadeh, Y. C. Chiu, E. M. Brey and A. Cinar, Tissue Eng., Part A, 2011, 17, 2133-2141.

43 A. V. Raghu, G. Anita, Y. M. Barigaddi, G. S. Gadaginamath and T. M. Aminabhavi, J. Appl. Polym. Sci., 2010, 104, 81-88. 44 R. Anjanapura, Polym. Eng. Sci., 2013, 54, 24-32. 